\title{
An Enhanced Light-weight Proactive Source Routing Protocol using BFS and DFS for MANET
}

\author{
Rashmi Dewangan $^{\# 1}$, Prof. Somesh Kumar Dewangan ${ }^{* 2}$ \\ ${ }^{\#}$ M.Tech, Department of CSE (Information Security) \\ Disha Institute of Management And Technology, Raipur, Chhattisgarh, India
}

\begin{abstract}
MANET is a self organized and self configurable network without existing infrastructure. It consists of several mobile wireless nodes. A routing protocol provides an efficient route between mobile nodes within the network. The discovery and maintenance of route should consume minimum overhead and bandwidth. In this paper, we introduce a novel Proactive Source Routing protocol that has a very small communication overhead. The proposed work is an efficient and improved light-weight proactive source routing protocol for MANETs that utilizes two common searching algorithms, called breadth first search (BFS) and depth first search (DFS) to discover the route. In such method, every node of the wireless network contains a neighbour table. Such table contains each node and its neighbours with distance between them. Therefore, each node has a full topology of the wireless network which is useful to discover the route. Periodic information exchange is used to update such table. We analyze the solution of routing in MANET and evaluate its performance using Network Simulator-2 (NS-2) under different network parameters.
\end{abstract}

Keywords-MANET, NS-2, Routing protocols, BFS, DFS, PSR, DSDV, AODV, OLSR, DSR.

\section{INTRODUCTION}

Mobile ad-hoc network (MANET) is a wireless communication network that contains various mobile devices. These mobile devices form a network with each other without any existing infrastructure or any other kind of fixed stations. It is a self-configuring and self organized network of mobile devices. These devices can move in any direction. The links between these devices will be change frequently, due to their movement. In a dynamic environment of the wireless network, nodes are independent and their mobility causes frequent change of network connectivity. Nodes in such network can act as end points of data transmission as well as routers when the two end points are not in direct range of each other. In a decentralized network, a node is responsible to find the topology information and deliverance of data to the destination. The implementation of appropriate routing protocol is based on the nature of application.

MANET continuously maintains the information required to properly route the traffic. MANET is a type of wireless adhoc network that usually has a routable networking environment on top of a Link Layer Ad-hoc network. The growth of laptops and Wi-Fi wireless networking has made mobile ad-hoc wireless networks a popular research topic. Various academic papers analyze routing protocols and their abilities, in the basis of varying degrees of mobility within a bounded space.

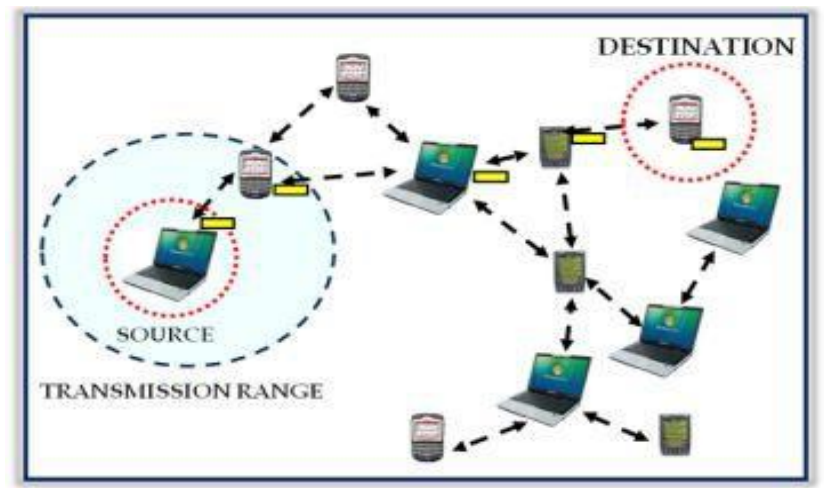

Fig 1: Mobile Ad-hoc Network

A mobile ad-hoc network (MANET) is a wireless communication network that can operate without existing infrastructure and support a number of mobile users. It is one of the general scopes of multi-hop wireless networking. Such networking paradigm originated from the needs in emergency operations, battlefield communications, search and rescue, and disaster relief operations. The main challenges in this area of research include end-to-end data forwarding, communication link access control, network security and providing support for real-time multimedia streaming. Centralized control and management or fixed network infrastructure such as base stations or access points are not essential in ad-hoc networks. Quick and inexpensive set up can be done for it, as needed. A mobile ad-hoc wireless network contains an autonomous group of mobile users that communicate over reasonably slow wireless links. Due to the mobility of nodes, many rapid and unpredictable changes may be done over the time. In such network, the mobile nodes maintain all the network activities like route discovery and message delivery, so that such network is decentralized.

In this paper, we propose a lightweight proactive source routing protocol to facilitate opportunistic data forwarding in MANETs. The information is periodically exchanged among neighbouring nodes for updated network topology 
information. Thus, it allows a node to have full-path information to all other nodes in the network. This allows it to support both source routing and conventional IP forwarding. When doing this, we try to reduce the routing overhead as much as we can. The results of simulation denote that our methodology has only a fraction of overhead of OLSR, DSDV, and DSR but still offers a similar or better data transportation capability compared with these protocols.

\section{ROUTING PROTOCOLS}

Routing is a process of sending a message from one mobile host in the network to another (it is also known as unicast). Routing protocols for ad-hoc wireless networks are generally used for mobility management and scalable design, in which mobility management is done through information exchanges between mobile hosts in the ad-hoc wireless network. Commonly, when information exchanges occur frequently, the network maintains accurate information of host locations and other relevant information. We know that frequent information exchange may be more expensive, since they consume more communication resources like bandwidth and power. With less frequent information exchanges, these costs diminish but there is more uncertainty about the host's location. Scalable design (one that works for large size networks) requires both routing protocols and resource consumptions to be scalable.

A routing protocol provides an efficient route between mobile nodes within the network. The discovery and maintenance of route should consume minimum overhead and bandwidth. Routing in the ad-hoc wireless network poses special challenges because of its infrastructure less network and its dynamic topology. The tunnel-based triangle routing of mobile IP works well if there is a fixed infrastructure to support the concept of the - home agent. However, when all hosts move (including the home agent), such a strategy cannot be directly applied. Traditional routing protocols for wired networks, such as link state or distance vector, are no longer suitable for mobile ad-hoc wireless networks. In such communication environment with mobile hosts as routers, convergence to new, stable routes after dynamic changes in network topology may be slow and this process could be costly due to low bandwidth. Routing information has to be localized to adapt quickly to changes such as hosts movement.

A routing protocol is essential whenever a packet needs to be handed over via several nodes to reach at its destination. A routing protocol has to discover a route for data packet delivery and make the packet delivered to the exact destination. Routing Protocols have been an active area of research for many years; many protocols have been suggested keeping applications and type of network in view. Routing Protocols in Ad-Hoc Networks can be classified into two types:

\section{A. Proactive or Table Driven Protocols}

This type of routing protocols is very familiar in fixed wired networks. In this approach, each ad-hoc node consists of a topology table, which contains the up to date networks nodes interaction information. This table is updated all the time and it gives the proactive protocols another name of table-driven. One or more routing tables are maintained at each node and are exchanged periodically to share the topology information with the neighboring nodes in order to maintain a consistent network view. Ad-hoc network based on proactive protocols, power and bandwidth consumption increased due to topology table exchange among nodes after each changing in nodes location. This takes place even if the network is in stand-by mode. The best network context for proactive protocols is the low (or no) mobility networks. The most accepted proactive protocols are DSDV and OLSR.

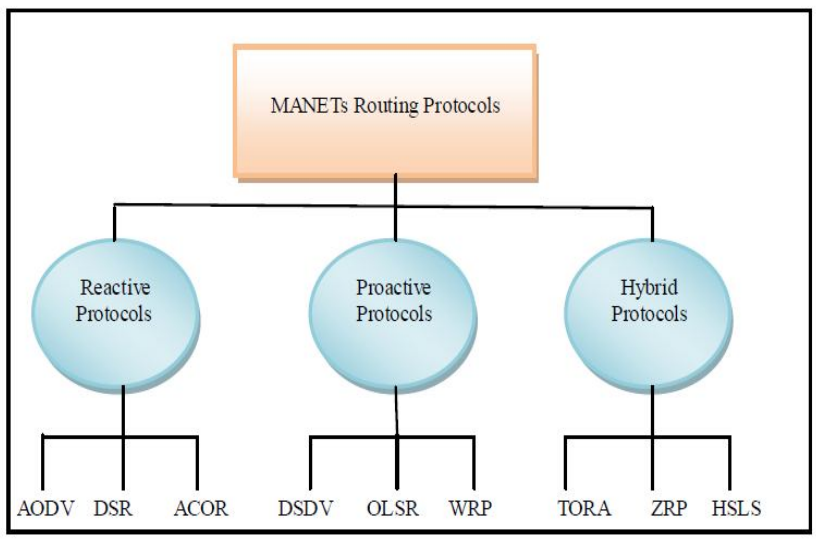

Fig 2: Routing Protocols in MANETs

\section{B. Reactive or On-demand Protocols}

Reactive routing techniques, also called on-demand routing, take different approach for routing than proactive protocols. Routes to the destination are discovered only when actually needed. When source node needs to send packet to some destination, it checks it routing table to determine whether it has a route. If no route exists, source node performs route discovery procedure to find a path to the destination. Reactive routing protocols can dramatically reduce routing overhead because they do not need to search for and maintain the routes on which there is no data traffic. Such property is so much important in the resource-limited environment. The most accepted reactive protocols are DSR and AODV. They do not initiate route discovery by themselves, until they are requested, when a source node request to find a route. These protocols setup routes when demanded. When a node wants to communicate with another node in the network, and the source node does not have a route to the node it wants to communicate with, reactive routing protocols will establish a route for the source to destination node.

\section{Hybrid Protocols}

Hybrid protocols inherit the advantage of high-speed routing form proactive and less overhead control messages from reactive protocols. The characteristics of proactive and reactive routing protocols can be integrated to achieve hybrid routing technique. Hybrid routing protocols may exhibit proactive or reactive behaviour depending on the 
circumstance, hence allow flexibility based on the wireless network. The most typical protocols are ZRP and TORA.

\section{RELATED WORK}

PSR proposed by Zehua Wang [2] maintains network topology using spanning tree. It takes the concept of BFS and streamlined differential update. Wang also propose a new concept, known as CORMAN [1], which is a network layer solution to the opportunistic data transfer in mobile ad-hoc networks. CORMAN contains three modules that provide a solution for one of the existing challenges.

Routing protocols in MANETs can be categorized using an array of criteria. The most fundamental among these is the timing of routing information exchange. On one hand, a protocol may require that nodes in the network should maintain valid routes to all destinations at all times. In this case, the protocol is considered proactive, which is also known as table driven. Examples of proactive routing protocols include destination sequenced distance vector (DSDV) [8] and OLSR [9]. On the other hand, if nodes in the network do not always maintain routing information, when a node receives data from the upper layer for a given destination, it must first find out about how to reach the destination. This approach is called reactive, which is also known as on demand. DSR [10] and ad-hoc on-demand DV (AODV) [11] fall in this category.

These well-known routing schemes can be also categorized by their fundamental algorithms. The most important algorithms in routing protocols are DV and LS algorithms. In an LS, every node floods the information of the links between itself and its neighbours in the entire network, such that every other node can reconstruct the complete topology of the network locally. In a DV, a node only provides its neighbours with the cost to reach each destination. With the estimates coming from neighbours, each node is able to determine which neighbour offers the best route to a given destination. Both LS and DV support the vanilla IP packets. DSR, however, takes a different approach to ondemand source routing. In DSR, a node employs a path search procedure when there is a need to send data to a particular destination. Once a path is identified by the returning search control packets, this entire path is embedded in each data packet to that destination. Thus, intermediate nodes do not even need a forwarding table to transfer these packets. Because of its reactive nature, it is more appropriate for occasional or lightweight data transportation in MANETs.

AODV, DSDV, and other DV-based routing algorithms were not designed for source routing; hence, they are not suitable for opportunistic data forwarding. The reason is that every node in these protocols only knows the next hop to reach a given destination node but not the complete path. OLSR and other LS-based routing protocols could support source routing, but their overhead is still fairly high for the load-sensitive MANETs. DSR and its derivations have a long bootstrap delay and are therefore not efficacious for frequent data exchange, particularly when there are a large number of data sources. In fact, many lightweight routing protocols had been proposed for the Internet to address its scalability issue, i.e., all naturally "table driven." The path-finding algorithm (PFA) is based on DVs and improves them by incorporating the predecessor of a destination in a routing update. Hence, the entire path to each node can be reconstructed by connecting the predecessors and destinations; therefore, the source node will have a tree topology rooted at itself. In the meantime, the link vector (LV) algorithm [35] reduces the overhead of LS algorithms to a great deal by only including links to be used in data forwarding in routing updates. The extreme case of LV, where only one link is included per destination, coincides with the PFA. PFA and LV were both originally proposed for the Internet, but their ideas were later used to devise routing protocols in the MANET. The Wireless Routing Protocol (WRP) [37] was an early attempt to port the routing capabilities of LS routing protocols to MANETs. It is built on the same framework of the PFA for each node to use a tree to achieve loop-free routing.

Although it is an innovative exploration in the research on MANETs, it has a rather high communication overhead due to the amount of information stored at and exchanged by the nodes. This is exacerbated by the same route update strategy as in the PFA, where routing updates are triggered by topology changes. Although this routing update strategy is reasonable for the PFA in the Internet, where the topology is relatively stable, this turns out to be fairly resource demanding in MANETs. (Our original intention was to include the WRP in the experimental comparison later in this paper, and we have implemented WRP in ns2. Unfortunately, our preliminary tests indicate that the changing topology in the MANET incurs an overwhelming amount of overhead, i.e., at least an order of magnitude higher than the other mainstream protocols. Thus, we do not include the simulation result of WRP as a baseline in our comparison.)

\section{METHODOLOGY}

This paper introduces a novel routing method to improve the performance of mobile ad-hoc networks, in which we develop an enhanced proactive source routing protocol for data transmission in such network. It diminishes the routing overhead and enhances the reliability of data transmission between the mobile nodes. This scheme achieves several objectives and challenges. To achieve our goal, some existing methods were used in our research. Such methodology improves the throughput and performance of MANET. Network simulator -2 (ns-2) is generally used in this research area by the research communities. NS-2 gives better result for mobile ad-hoc wireless networks.

Essentially, our method provides every node with a neighbour table for the entire network. To do that, nodes periodically broadcast the table information to their best knowledge in each iteration. Based on the information collected from neighbours during the most recent iteration, a node can refresh its knowledge about the network topology by adding such recent information. This knowledge will be distributed to its neighbours in the next round of operation. On the other hand, when a neighbour is deemed lost, a 
procedure is triggered to remove its relevant information from the topology repository maintained by the detecting node. Intuitively, the proposed scheme has about the same communication overhead as DV-based protocols. Differential update mechanism is also useful to reduce more routing overhead.

\section{1) Table Update}

Due to its proactive nature, the update operation of our work is iterative and distributed among all nodes in the network. At the beginning, node is only aware of the existence of itself. By exchanging the table information with the neighbours, it is able to maintain the network topology. In each subsequent iteration, nodes exchange their table data with their neighbours. From the perspective of source node, toward the end of each operation interval, it has received a set of routing messages from its neighbours. Note that, in fact, more nodes may be situated within the transmission range of source node, but their periodic updates were not received by it due to, for example, bad channel conditions. After all, the definition of a neighbour in MANETs is a fickle one. (We have more details on how we handle lost neighbours subsequently.) Source Node incorporates the most recent information from each neighbour to update its own table. It then broadcasts this information to its neighbours at the end of the period. In fact, in our implementation, the given update of the table happens multiple times within a single update interval so that a node can incorporate new route information to its knowledge base more quickly. This does not increase the communication overhead at all because one routing message is always sent per update interval.

TABLE I. NeIGHBour TABLE

\begin{tabular}{|c|c|c|c|c|c|c|}
\hline \multirow{2}{*}{$\begin{array}{c}\text { Node } \\
\text { ID }\end{array}$} & $\begin{array}{c}\text { Neighbour } \\
\text { ID }\end{array}$ & \multicolumn{2}{|c|}{$\begin{array}{c}\text { Node's } \\
\text { Position }\end{array}$} & \multicolumn{2}{c|}{$\begin{array}{c}\text { Neighbour's } \\
\text { Position }\end{array}$} & \multirow{2}{*}{ Distance } \\
\cline { 3 - 6 } & & $\mathbf{X}$ & $\mathbf{Y}$ & $\mathbf{X}$ & $\mathbf{Y}$ & \\
\hline 0 & 10 & 1063 & 41 & 1065 & 8 & 49 \\
6 & 7 & 392 & 39 & 579 & 187 & 20 \\
7 & 6 & 579 & 187 & 392 & 39 & 20 \\
9 & 11 & 1392 & 282 & 1362 & 226 & 58 \\
9 & 12 & 1385 & 195 & 1362 & 138 & 58 \\
10 & 0 & 1065 & 8 & 1063 & 41 & 49 \\
11 & 9 & 1362 & 226 & 1392 & 282 & 58 \\
& & & & & & \\
\hline
\end{tabular}

\section{2) Lost Neighbour Information Removal}

If a neighbour is disconnected from the network then each node removes all the data about the lost node. Such process is triggered by the following cases:

- No routing update or data packet has been received from this neighbour for a given time.

- A data transmission to such node has failed.

This process can be initiated more number of times.

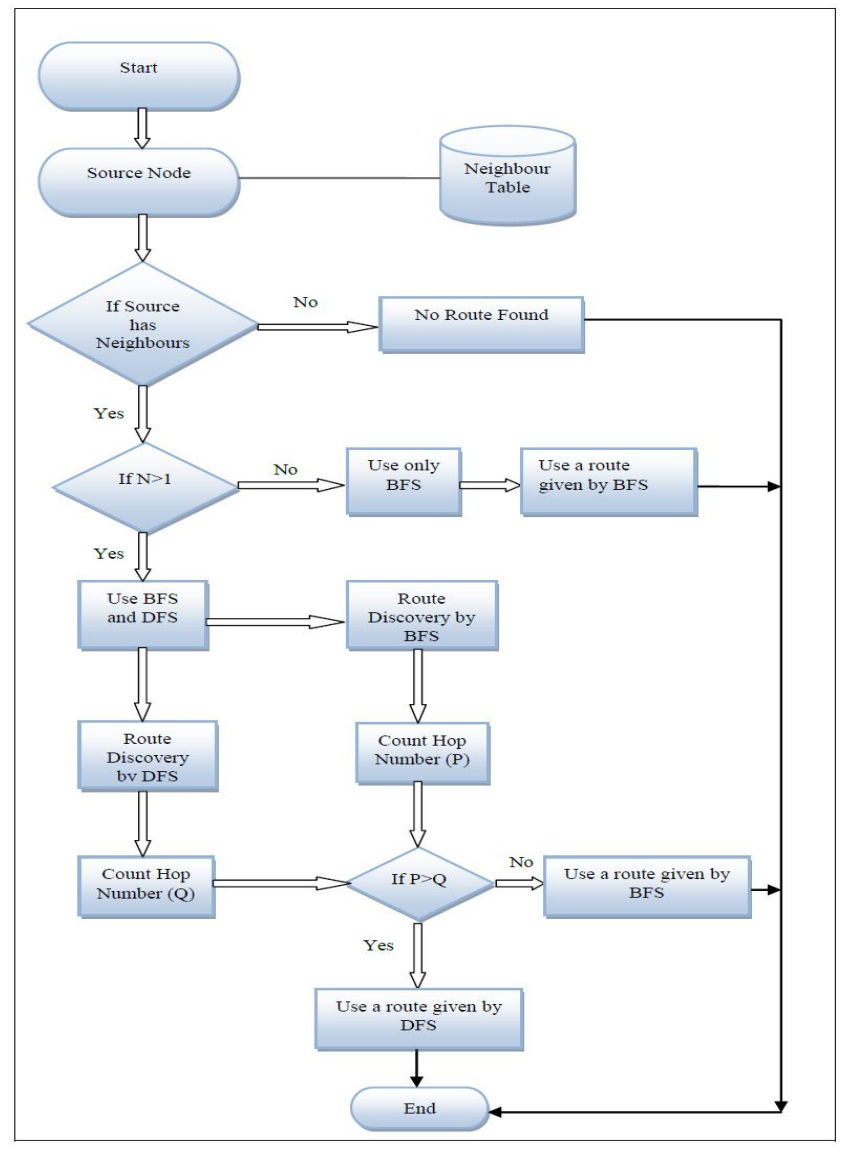

Fig 3: Data Flow Diagram

\section{3) Differential Update Mechanism}

In addition to dubbing route updates as hello messages in this mechanism, we interleave the "full dump" routing messages, with "differential updates". The basic idea is to send the full update messages less frequently than shorter messages containing the difference between the current and previous knowledge of a node's routing module. Our goal is to broadcast the information stored at a node to its neighbours in a short packet.

\section{4) Route Discovery using BFS and DFS}

The route discovery procedure is performed by Breadth First Search (BFS) and Depth First Search (DFS) in the wireless network. These search techniques work separately in the nodes of MANET. BFS and DFS algorithms are performed by two separate neighbour nodes of the source node. The optimized result is selected by such scheme and transfers the packet on the network. We can easily understand this process with Fig 3 that shows the data flow diagram.

\section{2) Use of Broadcasting Nature for Packet Transmission}

Broadcasting nature is a specific advantage of wireless network. The technique of ExOR is used in this paper. If source node sends a packet then some intermediate nodes also participate on such packet transfer. These intermediate 
nodes form a subset and perform an efficient data communication.

\section{PERFORMANCE EVALUATION}

We study the performance of our work using computer simulation with Network Simulator 2 (ns-2). C++ and Tool Command Language (TCL) are the two languages used in NS-2. It uses TCL/OTCL (Tool Command Language/ Object Oriented TCL) as a command \& configuration interface. Basically TCL is its scripting and frontend language and $\mathrm{C}++$ is its backend language. NS-2 includes a tool for viewing the simulation results, called Network Animator (NAM). It uses three types of files namely Tool Command Language file (.tcl), Trace file (.tr) and Network Animator file (.nam). Tool command language file (.tcl) has subsets of commands which are written into it for simulation. While simulator runs on .tcl, simulation trace file (.tr) and animation file (.nam) are created during the session. Trace file (.tr) is used to trace the whole process and Network Animator file (.nam) is used to visualize the behaviour of network protocols and traffic the model. We use various performance metrics required for evaluation of protocols. These matrices are important because it analyse the performance of the network.

We compare it against PSR, OLSR, DSDV, and DSR, in which OLSR, DSDV and DSR are three fundamentally different routing protocols in MANETs, with varying network densities and node mobility rates. Our tests show that the overhead of proposed method is indeed only a fraction of that of the baseline protocols. Nevertheless, as it provides global routing information at such a small cost, our method offers similar or even better data delivery performance. Here, we first describe how the experiment scenarios are configured and what measurements are collected.

TABLE II. SimULATION SETUP

\begin{tabular}{|c|c|}
\hline Simulation Parameters & Simulation Values \\
\hline Channel Type & Wireless channel \\
\hline Propagation model & Two-Ray Ground \\
\hline Network Interface Type & Phy/Wireless Phy \\
\hline Interface Queue Type & Queue/DropTail/PriQueue \\
\hline Transmission Range & $250 \mathrm{~m}$ \\
\hline Network Dimension & $1500 \mathrm{~m} * 750 \mathrm{~m}$ \\
\hline $\begin{array}{c}\text { Queue Capacity (in } \\
\text { packet) }\end{array}$ & 50 \\
\hline MAC Protocol & IEEE 802.11 \\
\hline Antenna Type & Omni antenna \\
\hline Simulation Time & 35 \\
\hline
\end{tabular}

Since many routing protocol's performances are well known in the classic two-ray ground reflection propagation model, we select such a model as well in our simulation to present a consistent and comparable result. With default physical-layer parameters of the simulator, the transmission range is approximately $250 \mathrm{~m}$. On such simulation, the simulation time is set on 35 in wireless channel.
Fig 4 shows a comparison graph for routing overhead with various node densities, in which PRO means proposed work.

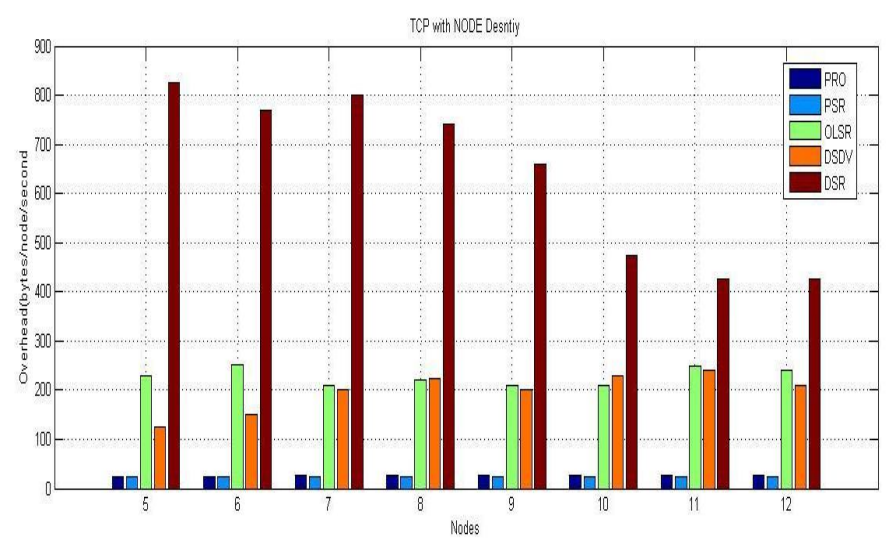

Fig 4: Routing Overhead Vs Node Density

We compare its performance with that of PSR, OLSR, DSDV, and DSR. The reasons that we select these baseline protocols that are different in nature are as follows. On one hand, PSR, OLSR and DSDV are proactive routing protocols, and our technique is also in this category. On the other hand, OLSR makes complete topological structure available at each node, whereas in DSDV, nodes only have distance estimates to other nodes via a neighbour. PSR sits in the middle ground, where each node maintains a spanning tree of the network. Furthermore, DSR is a well-accepted reactive source routing scheme, and as with our method, it support source routing. All three baseline protocols and PSR are configured and tested out of the box of ns-2. Here, different graphs indicated different performance metrics and analysis of our method with some other routing protocols.

Fig 5 shows a comparison graph for end-to-end delay with various node densities.

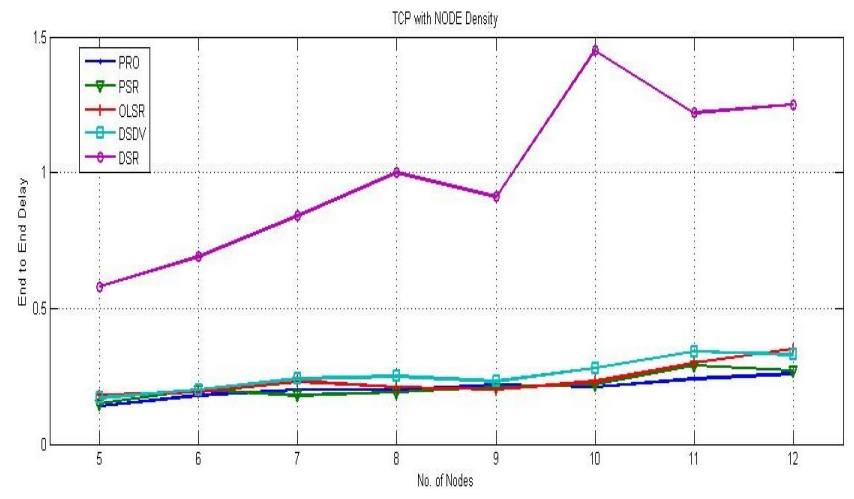

Fig 5: End-to-end Delay Vs Node Density

Fig 6 shows a comparison graph for path estimation effort between PSR and proposed work. 


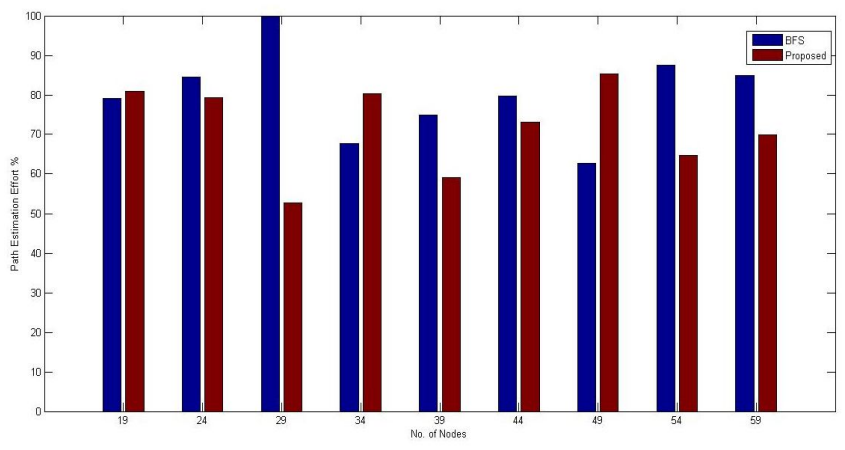

Fig 6: Path Estimation Effort (\%) Vs Number of Nodes

\section{CONCLUSION}

This paper has been motivated by the need to support opportunistic data forwarding in MANETs. To generalize the milestone work of ExOR for it to function in such networks, we needed an efficient proactive protocol. Such a protocol should provide more topology information than DVs but must have significantly smaller overhead than LS routing protocols; even the MPR technique in OLSR would not suffice. Thus, we put forward a table-based routing protocol using BFS and DFS. Its routing overhead per time unit per node is on the order of the number of the nodes in the network as with DSDV, but each node has the full-path information to reach all other nodes. Proposed method uses only one type of message, i.e., the periodic data update, both to exchange routing information and as hello beacon messages. We interleave full-dump messages with differential updates so that, in relatively stable networks, the differential updates are much shorter than the full-dump messages. As a result, the routing overhead of proposed work is only a fraction or less compared with DSDV, OLSR, and DSR, as evidenced by our experiments. It is better than PSR. In the simulation in this paper, we used such method to support traditional IP forwarding for a closer comparison with DSDV and OLSR, whereas DSR still carried sourcerouted messages. In our work, we tested the capability in transporting source-routed packets for opportunistic data forwarding, where we also found that its small overhead met our initial goal. This is fundamentally different from traditional IP forwarding in proactive routing with more built-in adaptivity, where the routing information maintained at nodes closer to the destination is often more updated than the source node.

\section{ACKNOWLEDGEMENT}

I am very much grateful to Department of CSE, DIMAT to give me opportunity to work on Routing in Mobile Ad-hoc Networks. I sincerely express my gratitude to Prof. Somesh Kumar Dewangan, Dept. of CSE, DIMAT for giving constant inspiration for this work. I am also thankful to Mrs. Preeti Tuli, Dept. of CSE, DIMAT for helping me directly and indirectly during this work. I am really thankful to my all friends for their blessing and support.

\section{REFERENCES}

[1] Z. Wang, Y. Chen, and C. Li, "CORMAN: a Novel Cooperative Opportunistic Routing Scheme in Mobile Ad Hoc Networks," IEEE Journal on Selected Areas In Communications, Vol. 30, No. 2, February 2012, pp 289-296.

[2] Z. Wang, C. Li, and Y. Chen, "PSR: Proactive Source Routing in Mobile Ad Hoc Networks," in Proceedings of the 2011 IEEE Conference on Global Telecommunications (GLOBECOM), Houston, TX USA, December 2011.

[3] Z. Wang, Y. Chen, and C. Li, "A New Loop-Free Proactive Source Routing Scheme for Opportunistic Data Forwarding in Wireless Networks," IEEE Communications in 2012.

[4] Z. Wang, Y. Chen, and C. Li, "PSR: A Light-Weight Proactive Source Routing Protocol for Mobile Ad Hoc Networks," in IEEE Transactions on Vehicular Technology, Vol. 63, No. 2, February 2014.

[5] Z. Wang, C. Li, and Y. Chen, "Local Cooperative Retransmission in Opportunistic Data Forwarding," in 2012 IEEE International Conference on Communications (ICC).

[6] I. Chlamtac, M. Conti, and J.-N. Liu, "Mobile Ad hoc Networking: Imperatives and Challenges," Ad Hoc Networks, vol. 1, no. 1, pp. 1364, July 2003.

[7] R. Rajaraman, "Topology control and routing in ad hoc networks: A survey," SIGACT News, vol. 33, pp. 60-73, June 2002

[8] C. E. Perkins and P. Bhagwat, "Highly Dynamic DestinationSequenced Distance-Vector Routing (DSDV) for Mobile Computers," Computer Communication Review, pp. 234-244, October 1994.

[9] T. Clausen and P. Jacquet, "Optimized Link State Routing Protocol (OLSR)," RFC 3626, October 2003. [Online]. Available: http://www.ietf.org/rfc/rfc3626.txt

[10] D. B. Johnson, Y.-C. Hu, and D. A. Maltz, "On The Dynamic Source Routing Protocol (DSR) for Mobile Ad Hoc Networks for IPv4,” RFC 4728, February 2007. [Online]. Available: http://www.ietf.org/rfc/rfc4728.txt

[11] C. E. Perkins and E. M. Royer, "Ad hoc On-Demand Distance Vector (AODV) Routing," RFC 3561, July 2003. [Online]. Available: http://www.ietf.org/rfc/rfc3561.txt

[12] T. M. Cover and A. A. E. Gamal, "Capacity Theorems for the Relay Channel," IEEE Transactions on Information Theory, vol. 25, no. 5, pp. 572-584, September 1979.

[13] A. Nosratinia, T. E. Hunter, and A. Hedayat, "Cooperative Communication in Wireless Networks," IEEE Communications Magazine, vol. 42, no. 10, pp. 74-80, October 2004.

[14] S. Biswas and R. Morris, "ExOR: Opportunistic Multi-Hop Routing for Wireless Networks," in Proceedings of ACM Conference of the Special Interest Group on Data Communication (SIGCOMM), Philadelphia, PA, USA, August 2005, pp. 133-144.

[15] P. Larsson, "Selection Diversity Forwarding in a Multihop Packet Radio Network With Fading Channel and Capture," ACM Mobile Computing and Communications Review, vol. 5, no. 4, pp. 47-54, October 2001

[16] E. Rozner, J. Seshadri, Y. Mehta, and L. Qiu, "Simple Opportunistic Routing Protocol for Wireless Mesh Networks," in Proceedings of the 2nd IEEE Workshop on Wireless Mesh Networks (WiMesh), Sep. 2006, pp. $48-54$.

[17] M. Kurth, A. Zubow, and J.-P. Redlich, "Cooperative Opportunistic Routing Using Transmit Diversity in Wireless Mesh Networks," in Proceedings of the $27^{\text {th }}$ IEEE International Conference on Computer Communication (INFOCOM), Apr. 2008, pp. 1310-1318.

[18] M. Naghshvar and T. Javidi, "Opportunistic Routing with Congestion Diversity in Wireless Multi-hop Networks," in Proceedings of the 29th IEEE International Conference on Computer Communication (INFOCOM). Piscataway, NJ, USA: IEEE Press, 2010, pp. 496-500.

[19] K. Zeng, Z. Yang, and W. Lou, "Opportunistic Routing in Multi-Radio Multi-Channel Multi-Hop Wireless Networks," in Proceedings of the 29th IEEE International Conference on Computer Communication (INFOCOM). Piscataway, NJ, USA: IEEE Press, 2010, pp. 476-480.

[20] S. Yang, F. Zhong, C. K. Yeo, B. S. Lee, and J. Boleng, "Position Based Opportunistic Routing for Robust Data Delivery in MANETs," in Proceedings of the 2009 IEEE Conference on Global 
Telecommunications (GLOBECOM), Honolulu, Hawaii, USA, December 2009, pp. 1325-1330.

[21] Z. Zhong and S. Nelakuditit, "On the Efficacy of Opportunistic Routing," in Proceedings of the 4th IEEE Communications Society Conference on Sensor, Mesh and Ad Hoc Communications and Networks (SECON), Jun. 2007, pp. 441-450.

[22] S. Chachulski, M. Jennings, S. Katti, and D. Katabi, "Trading Structure for Randomness in Wireless Opportunistic Routing," in Proceedings of ACM Conference of the Special Interest Group on Data Communication (SIGCOMM), Kyoto, Japan, August 2007, pp. 169180.

[23] D. S. J. De Couto, D. Aguayo, J. Bicket, and R. Morris, "A HighThroughput Path Metric for Multi-Hop Wireless Routing," in Proceedings of the 9th Annual International Conference on Mobile Computing and Networking (MobiCom), San Diego, CA, USA, 2003, pp. 134-146.

[24] J. Ma, Q. Zhang, C. Qian, and L. M. Ni, "Energy-Efficient Opportunistic Topology Control in Wireless Sensor Networks," in Proceedings of the 1st International MobiSys Workshop on Mobile Opportunistic Networking (MobiOpp). New York, NY, USA: ACM, 2007, pp. 33-38.

[25] I. Leontiadis and C. Mascolo, "GeOpps: Geographical Opportunistic Routing for Vehicular Networks," in Proceedings of the IEEE International Symposium on a World of Wireless Mobile and Multimedia Networks (WoWMoM), Helsinki, Finland, June 2007, pp. $1-6$.

[26] B. Radunović, C. Gkantsidis, P. Key, and P. Rodriguez, "An Optimization Framework for Opportunistic Multipath Routing in Wireless Mesh Networks," in Proceedings of the 27th IEEE International Conference on Computer Communication (INFOCOM), Apr. 2008, pp. 2252-2260.

[27] D. Koutsonikolas, C.-C. Wang, and Y. C. Hu, "CCACK: Efficient Network Coding Based Opportunistic Routing through Cumulative Coded Acknowledgments," in Proceedings of the 29th IEEE International Conference on Computer Communication (INFOCOM). Piscataway, NJ, USA: IEEE Press, 2010, pp. 2919-2927.

[28] R. C. Shah, S. Wietholter, A.Wolisz, and J. M. Rabaey, "When does Opportunistic Routing Make Sense?"' in Proceedings of the 3rd Annual IEEE International Conference on Pervasive Computing and Communications (PerCom), Mar. 2005, pp. 350-356.

[29] J. Kim and S. Bohacek, "A Comparison of Opportunistic and Deterministic Forwarding in Mobile Multihop Wireless Networks," in Proceedings of the 1st International MobiSys Workshop on Mobile Opportunistic Networking (MobiOpp). New York, NY, USA: ACM, 2007, pp. 9-16.
[30] K. Zeng, W. Lou, and H. Zhai, "On End-to-End Throughput of Opportunistic Routing in Multirate and Multihop Wireless Networks," in Proceedings of the 27th IEEE International Conference on Computer Communication (INFOCOM), Apr. 2008, pp. 816-824.

[31] L. Cerda-Alabern, V. Pla, and A. Darehshoorzadeh, "On the Performance Modeling of Opportunistic Routing," in Proceedings of the 2nd International Workshop on Mobile Opportunistic Networking (MobiOpp). New York, NY, USA: ACM, 2010, pp. 15-21.

[32] M.-H. Lu, P. Steenkiste, and T. Chen, "Video Transmission over Wireless Multihop Networks Using Opportunistic Routing," in Proceedings of the 16th IEEE International Packet Video Workshop (PV), Nov. 2007, pp. 52-61.

[33] F. Wu, T. Chen, S. Zhong, L. E. Li, and Y. R. Yang, "IncentiveCompatible Opportunistic Routing for Wireless Networks," in Proceedings of the 14th ACM International Conference on Mobile Computing and Networking (MobiCom). New York, NY, USA: ACM, 2008, pp. 303-314.

[34] J. J. Garcia-Luna-Aceves and S. Murthy, "A Path-Finding Algorithm for Loop-Free Routing," IEEE/ACM Transactions on Networking, vol. 5, pp. 148-160, February 1997.

[35] J. Behrens and J. J. Garcia-Luna-Aceves, "Distributed, Scalable Routing based on Link-State Vectors," in Proceedings of ACM SIGCOMM, 1994, pp. 136-147.

[36] K. Levchenko, G. M. Voelker, R. Paturi, and S. Savage, "XL: An Efficient Network Routing Algorithm," in Proceedings of ACM SIGCOMM, 2008, pp. 15-26.

[37] S. Murthy and J. J. Garcia-Luna-Aceves, "An Efficient Routing Protocol for Wireless Networks," Mobile Networks and Applications, vol. 1, no. 2, pp. 183-197, October 1996.

[38] J. J. Garcia-Luna-Aceves and M. Spohn, "Source-Tree Routing in Wireless Networks," in Proceedings of the 7th Annual International Conference on Network Protocols (ICNP'99), Toronto, Canada, October 1999, pp. 273-282.

[39] X. Yu, "Distributed Cache Updating for the Dynamic Source Routing Protocol," IEEE Transactions on Mobile Computing, vol. 5, no. 6, pp. 609-626, June 2006.

[40] Y.-C. Hu and D. B. Johnson, "Implicit Source Routes for On-Demand Ad Hoc Network Routing," in Proceedings of the 3rd ACM International Symposium on Mobile Ad Hoc Networking and Computing (MobiHoc'01), Long Beach, CA, USA, October 2001, pp. $1-10$.

[41] B. Hu and H. Gharavi, "DSR-Based Directional Routing Protocol for Ad Hoc Networks," in Proceedings of the 2007 IEEE Conference on Global Telecommunications (GLOBECOM), Washington D.C., DC USA, November 2007, pp. 4936-4940. 